\title{
Knowledge Discovery in Medical Multi-databases: A Rough Set Approach
}

\author{
Shusaku Tsumoto \\ Department of Medicine Informatics, Shimane Medical University, School of Medicine, \\ 89-1 Enya-cho Izumo City, Shimane 693-8501 Japan \\ E-mail: tsumoto@computer.org
}

\begin{abstract}
Since early 1980's, due to the rapid growth of hospital information systems (HIS), electronic patient records are stored as huge databases at many hospitals. One of the most important problems is that the rules induced from each hospital may be different from those induced from other hospitals, which are very difficult even for medical experts to interpret. In this paper, we introduce rough set based analysis in order to solve this problem. Rough set based analysis interprets the conflicts between rules from the viewpoint of supporting sets, which are closely related with dempster-shafer theory(evidence theory) and outputs interpretation of rules with evidential degree. The proposed method was evaluated on two medical databases, the experimental results of which show that several interesting relations between rules, including interpretation on difference and the solution of conflicts between induced rules, are discovered.
\end{abstract}

\section{Introduction}

Since early 1980's, due to the rapid growth of hospital information systems (HIS), electronic patient records are stored as huge databases at many hospitals. One of the most important problems is that the rules induced from each hospital may be different from those induced from other hospitals, which are very difficult even for medical experts to interpret.

In this paper, we introduce rough set based analysis in order to solve this problem. Rough set based analysis interprets the conflicts between rules from the viewpoint of supporting sets, which are closely related with dempster-shafer theory(evidence theory) and outputs interpretation of rules with evidential degree. The proposed method was evaluated on two medical databases, the experimental results of which show that several interesting relations between rules, including interpretation on difference and the solution of conflicts between induced rules, are discovered.

The paper is organized as follows: Section 2 will make a brief description about distributed data analysis. Section 3 and 4 discusses the definition of rules and rough set model of distributed data analysis. Section 5 gives experimental results. Section 6 discusses the problems of our work and related work, and finally, Section 7 concludes our paper. 


\section{Distributed Data Analysis}

In distributed rule induction, the following three cases should be considered. (1) One database induces rules, whose attribute-value pairs do not appear in other database(independent type). (2) Rules induced from one database are overlapped with rules induced from other databases(boundary type). (3) Rules induced from one database are described by the subset of attribute-value pairs, which are used in rules induced from other databases(subcategory type). In the first case, it would be very difficult to interpret all the results because each database do no share the regularities with other databases. In the second case, shared information will be much more important than other information. In the third case, subset information will be important. It is notable that this classification on distributed data analysis can be applied to discussion on collaboration between domain experts and rule discovery methods: Empirical studies on medical data mining 211 show that medical experts try to interpret unexpected patterns with their domain knowledge, which can be viewed as hypothesis generation. In [2], gender is an attribute unexpected by experts, which led to a new hypothesis that body size will be closely related with complications of angiography. In [11,12, gender and age are unexpected attributes, which triggered reexamination of datasets and generated a hypothesis that immunological factors will be closely related with meningitis.

These actions will be summerized into the following three patterns:

1. If induced patterns are completely equivalent to domain knowledge, then the patterns are commonsense.

2. If induced patterns partially overlap with domain knowledge, then the patterns may include unexpected or interesting subpatterns.

3. If induced patterns are completely different from domain knowledge, then the patterns are difficult to interpret.

Then, the next step will be validation of a generated hypothesis: a dataset will be collected under the hypothesis in a prospective way. After the data collection, statistical analysis will be applied to detect the significance of this hypothesis. If the hypothesis is confirmed with statistical significance, these results will be reported. Thus, such a kind of interaction between human experts and rule discovery methods can be viewed as distributed data analysis.

\section{Probabilistic Rules}

\subsection{Accuracy and Coverage}

In the subsequent sections, we adopt the following notations, which is introduced in [8].

Let $U$ denote a nonempty, finite set called the universe and A denote a nonempty, finite set of attributes, i.e., $a: U \rightarrow V_{a}$ for $a \in A$, where $V_{a}$ is called the domain of $a$, respectively.Then, a decision table is defined as an information 
system, $A=(U, A \cup\{d\})$. The atomic formulas over $B \subseteq A \cup\{d\}$ and $V$ are expressions of the form $[a=v]$, called descriptors over $\mathrm{B}$, where $a \in B$ and $v \in V_{a}$. The set $F(B, V)$ of formulas over B is the least set containing all atomic formulas over $B$ and closed with respect to disjunction, conjunction and negation. For each $f \in F(B, V), f_{A}$ denote the meaning of $f$ in $A$, i.e., the set of all objects in $\mathrm{U}$ with property $f$, defined inductively as follows.

1. If $f$ is of the form $[a=v]$ then, $f_{A}=\{s \in U \mid a(s)=v\}$

2. $(f \wedge g)_{A}=f_{A} \cap g_{A} ;(f \vee g)_{A}=f_{A} \vee g_{A} ;(\neg f)_{A}=U-f_{a}$

By the use of this framework, classification accuracy and coverage, or true positive rate is defined as follows.

\section{Definition 1.}

Let $R$ and $D$ denote a formula in $F(B, V)$ and a set of objects which belong to a decision $d$. Classification accuracy and coverage(true positive rate) for $R \rightarrow d$ is defined as:

$$
\alpha_{R}(D)=\frac{\left|R_{A} \cap D\right|}{\left|R_{A}\right|}(=P(D \mid R)), \text { and } \kappa_{R}(D)=\frac{\left|R_{A} \cap D\right|}{|D|}(=P(R \mid D)),
$$

where $|A|$ denotes the cardinality of a set $A, \alpha_{R}(D)$ denotes a classification accuracy of $R$ as to classification of $D$, and $\kappa_{R}(D)$ denotes a coverage, or a true positive rate of $R$ to $D$, respectively.

It is notable that these two measures are equal to conditional probabilities: accuracy is a probability of $D$ under the condition of $R$, coverage is one of $R$ under the condition of $D$.

\subsection{Definition of Rules}

By the use of accuracy and coverage, a probabilistic rule is defined as:

$$
R \stackrel{\alpha, \kappa}{\rightarrow} d \quad \text { s.t. } \quad R=\wedge_{j} \vee_{k}\left[a_{j}=v_{k}\right], \alpha_{R}(D) \geq \delta_{\alpha}, \kappa_{R}(D) \geq \delta_{\kappa} .
$$

This rule is a kind of probabilistic proposition with two statistical measures, which is an extension of Ziarko's variable precision model(VPRS) 14] 1

\section{Rough Set Model of Distributed Data Analysis}

\subsection{Definition of Characterization Set}

In order to model these three reasoning types, a statistical measure, coverage $\kappa_{R}(D)$ plays an important role in modeling, which is a conditional probability of a condition $(R)$ under the decision $D(P(R \mid D))$.

\footnotetext{
1 This probabilistic rule is also a kind of Rough Modus Ponens 6 .
} 
Let us define a characterization set of $D$, denoted by $L(D)$ as a set, each element of which is an elementary attribute-value pair $R$ with coverage being larger than a given threshold, $\delta_{\kappa}$. That is,

$$
L_{\delta_{\kappa}}(D)=\left\{\left[a_{i}=v_{j}\right] \mid \kappa_{\left[a_{i}=v_{j}\right]}(D)>\delta_{\kappa}\right\}
$$

Then, according to the descriptions in section 2, three types of differences will be defined as below:

1. Independent type: $L_{\delta_{\kappa}}\left(D_{i}\right) \cap L_{\delta_{\kappa}}\left(D_{j}\right)=\phi$,

2. Boundary type: $L_{\delta_{\kappa}}\left(D_{i}\right) \cap L_{\delta_{\kappa}}\left(D_{j}\right) \neq \phi$, and

3. Subcatgory type: $L_{\delta_{\kappa}}\left(D_{i}\right) \subseteq L_{\delta_{\kappa}}\left(D_{j}\right)$,

where $i$ and $j$ denotes a table $i$ and $j$. All three definitions correspond to the negative region, boundary region, and positive region[4], respectively, if a set of the whole elementary attribute-value pairs will be taken as the universe of discourse. Thus, here we can apply the technique which is similar to inductiong of decision rules from the partition of equivalence relations. In the cases of boundary and subcategory type, the lower and upper limits of characterization are defined as:

$$
\begin{aligned}
& \underline{L}_{\delta_{k} a p p a}(D)=\cap_{i} L_{\delta_{k} a p p a}\left(D_{i}\right) \\
& \bar{L}_{\delta_{k} a p p a}(D)=\cup_{i} L_{\delta_{k} a p p a}\left(D_{i}\right)
\end{aligned}
$$

Concerning independent type, the lower limit is empty: $\bar{L}_{\kappa}(D)=$ and only the upper limit of characterization is defined. The lower limit of characterization is a set whose elements are included in all the databases, which can be viewed as information shared by all the datasets. The upper limit of characterization is a set whose elements are included in at least one database, which can be viwed as possible information shared by datasets. It is notable that the size of those limits is dependent on the choice of the threshold $\delta_{\kappa}$.

\subsection{Characterization as Exclusive Rules}

Characteristics of characterization set depends on the value of $\delta_{\kappa}$. If the threshold is set to 1.0, then a characterization set is equivalent to a set of attributes in exclusive rules 9]. That is, the meaning of each attribute-value pair in $L_{1.0}(D)$ covers all the examples of $D$. Thus, in other words, some examples which do not satisfy any pairs in $L_{1.0}(D)$ will not belong to a class $D$. Construction of rules based on $L_{1.0}$ are discussed in Subsection 4.4, which can also be found in [1012]. The differences between these two papers are the following: in the former paper, independent type and subcategory type for $L_{1.0}$ are focused on to represent diagnostic rules and applied to discovery of decision rules in medical databases. On the other hand, in the latter paper, a boundary type for $L_{1.0}$ is focused on and applied to discovery of plausible rules. 


\subsection{Rough Inclusion}

Concerning the boundary type, it is important to consider the similarities between classes. In order to measure the similarity between classes with respect to characterization, we introduce a rough inclusion measure $\mu$, which is defined as follows:

$$
\mu(S, T)=\frac{|S \bigcap T|}{|S|} .
$$

It is notable that if $S \subseteq T$, then $\mu(S, T)=1.0$, which shows that this relation extends subset and superset relations. This measure is introduced by Polkowski and Skowron in their study on rough mereology [7, which focuses on set-inclusion to characterize a hierarchical structure based on a relation between a subset and superset. Thus, application of rough inclusion to capturing the relations between classes is equivalent to constructing rough hierarchical structure between classes, which is also closely related with information granulation proposed by Zadeh [13].

\section{An Algorithm for Analysis}

An algorithms for searching for the lower and upper limit of characterization and induction of rules based on these limits are given in Fig. 1 and Fig. 2. Since subcategory type and independent type can be viewed as special types of boundary type with respect to rough inclusion, rule induction algorithms for subcategory type and independent type are given if the thresholds for $\mu$ are set up to 1.0 and 0.0 , respectively.

Rule discovery(Fig 1.) consists of the following three procedures. First, the characterization of each given class is extracted from each database and the lower and upper limit of characterization is calculated. Second, from these limits, rule induction method(Fig.2) will be applied. Finally, all the characteriztion are classified into several groups with respect to rough inclusion and the degree of similarity will be output.

\section{Experimental Results}

\subsection{Applied Datasets}

For experimental evaluation, a new system, called PRIMEROSE-REX5 (Probabilistic Rule Induction Method for Rules of Expert System ver 5.0), is developed with the algorithms discussed above. PRIMEROSE-REX5 was applied to the following three medical domains, whose information is shown in Table 1 


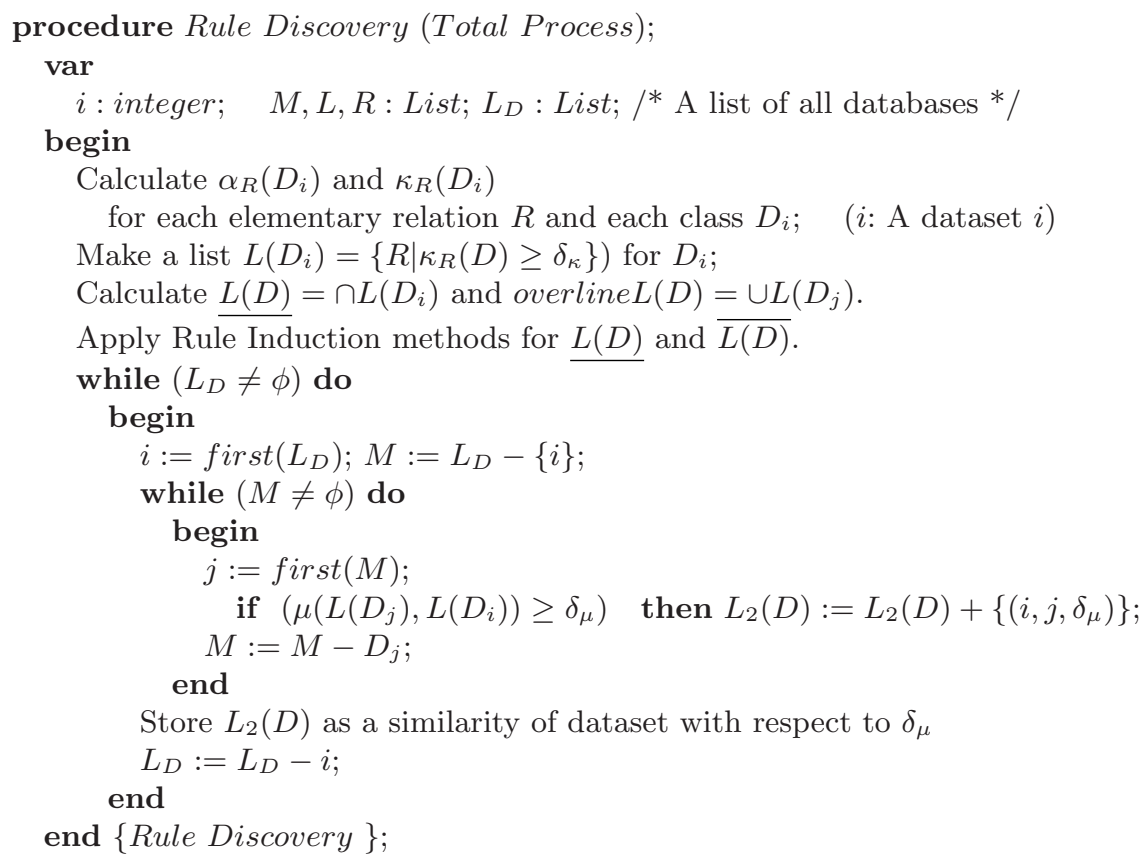

Fig. 1. An Algorithm for Rule Discovery

Table 1. Databases

\begin{tabular}{lrrrr} 
Domain & Tables Samples & Classes & Attributes \\
\hline Headache & 10 & 52119 & 45 & 147 \\
CVD & 4 & 7620 & 22 & 285 \\
Meningitis & 5 & 1211 & 4 & 41 \\
\hline
\end{tabular}

\subsection{Discovery in Experiments}

Characterization of Headache. Although all the rules from the lower and upper limit were not interesting for domain experts, several interesting and unexpected relations on the degree of similarity were found in characterization sets. Ten hospitals are grouped in three groups. Table 2 shows several information about these groups, each differentiated factor of which are regions. The first group is mainly located on the countryside, most of the people are farmers. The second one is mainly located in the housing area. Finally, the third group is in the business area.

Those groups included several interesting features for differential diagnosis of headache. In the first group, hypertension was one of the most important attributes for differential diagnosis. In the housing area, the nature of headache 


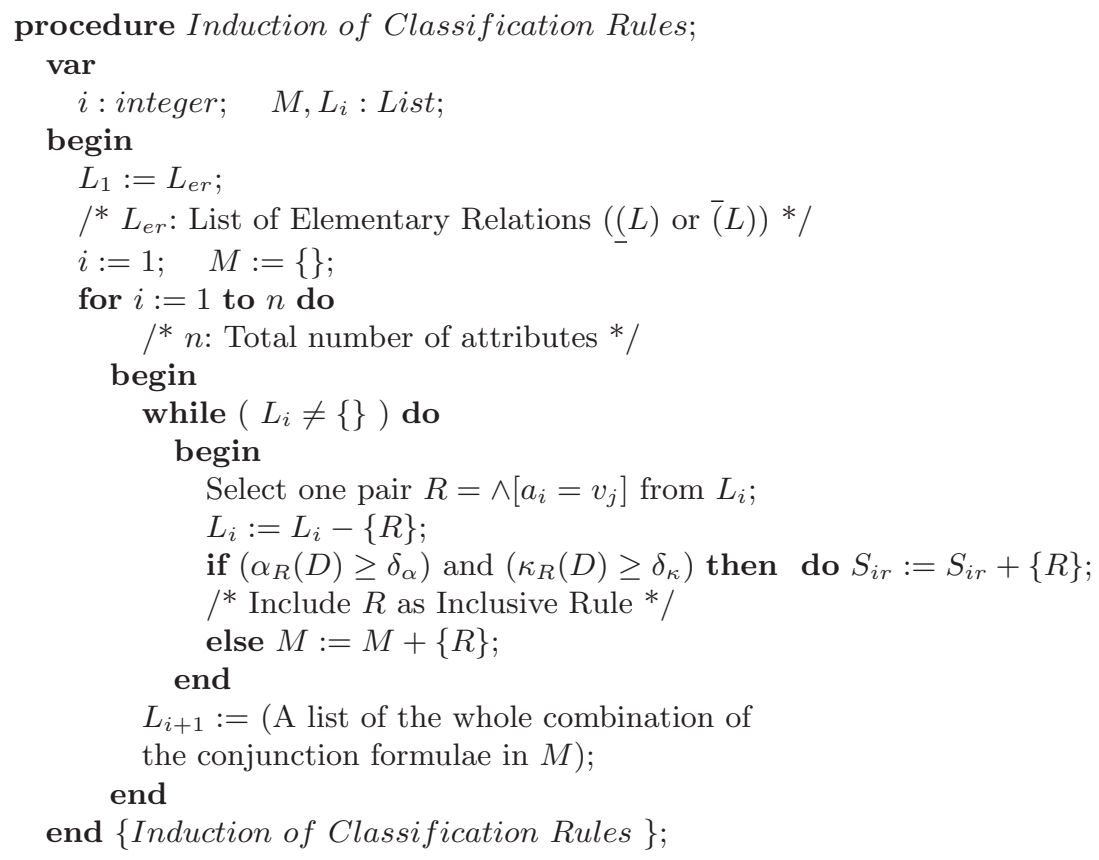

Fig. 2. An Algorithm for Classification Rules

was important for differential diagnosis. Finally, in the business area, the location of headache was important. According to domain experts' comments, these attributes are closely related with working environments.

This analysis suggests that the differences in upper limit and lower limit also include information, which lead to knowledge discovery.

Table 2. Characterization in Headache

\begin{tabular}{lcc}
\multicolumn{2}{c}{ Location } & Important Features in Upper Limit \\
\hline G1 Countryside & Hypertension $=$ yes \\
G2 & Housing & Nature $=$ chronic, acute \\
G3 & Business & Location $=$ neck,occipital \\
\hline
\end{tabular}

Rules of CVD. Concerning the database on CVD, several interesting rules are derived both from the lower limit and the upper limit. The most interesting results of lower limit are the following rules for thalamus hemorrahge:

$$
\begin{aligned}
& {[\text { Sex }=\text { Female }] } \wedge[\text { Hemiparesis }=\text { Left }] \wedge[\text { LOC }: \text { positive }] \rightarrow \text { Thalamus } \\
& \neg[\text { Risk }: \text { Hypertension }] \wedge \neg[\text { Sensory }=\text { no }] \rightarrow \neg \text { Thalamus }
\end{aligned}
$$


Interestingly, LOC(loss of consciousness) under the condition of $[$ Sex $=$ Female $] \wedge[$ Hemiparesis $=$ Left $]$ is an important factor to diagnose thalamic damage. In this domain, any strong correlations between these attributes and others, like the database of meningitis, have not been found yet. It will be our future work to find what factor will be behind these rules.

Rules of Meningitis. In the domain of meningitis, the following rules from the lower limit of charcacterization, which medical experts do not expect, are obtained.

$$
\begin{array}{r}
{[W B C<12000] \wedge[\text { Sex }=\text { Female }] \wedge[\text { Age }<40] \wedge\left[C S F \_C E L L<1000\right]} \\
\\
\rightarrow \text { Virus } \\
{[\text { Age } \geq 40] \wedge[W B C \geq 8000] \wedge[\text { Sex }=\text { Male }] \wedge\left[C S F \_C E L L \geq 1000\right]} \\
\rightarrow \text { Bacteria }
\end{array}
$$

The most interesting points are that these rules have information about age and sex, which often seems to be unimportant attributes for differential diagnosis.

The first discovery is that women do not often suffer from bacterial infection, compared with men, since such relationships between sex and meningitis has not been discussed in medical context 1]. Examined the database of meningitis closely, it is found that most of the above patients suffer from chronic diseases, such as DM, LC, and sinusitis, which are the risk factors of bacterial meningitis. The second discovery is that $[$ age $<40]$ is also an important factor not to suspect viral meningitis, which also matches the fact that most old people suffer from chronic diseases.

These results were also re-evaluted in medical practice. Recently, the above two rules were checked by additional 21 cases who suffered from meningitis (15 cases: viral and 6 cases: bacterial meningitis ) at a hospital which is different from the hospitals where datasets were collected. Surprisingly, the above rules misclassfied only three cases (two are viral, and the other is bacterial), that is, the total accuracy is equal to $18 / 21=85.7 \%$ and the accuracies for viral and bacterial meningitis are equal to $13 / 15=86.7 \%$ and $5 / 6=83.3 \%$. The reasons of misclassification are the following: a case of bacterial infection is a patient who have a severe immunodeficiency, although he is very young. Two cases of viral infection are patients who also have suffered from herpes zoster. It is notable that even those misclassficiation cases can be explained from the viewpoint of the immunodeficiency: that is, it is confirmed that immunodefiency is a key word for menigitis. The validation of these rules is still ongoing, which will be reported in the near future.

\section{Discussion: Conflict Analysis}

It is easy to see the relations of independent type and subcategory type. While independent type suggests different mechanisms of diseases, subcategory type 
does the same etiology. The difficult one is boundary type, where several symptoms are overlapped in each $L_{\delta_{\kappa}}(D)$. In this case, relations between $L_{\delta_{\kappa}}\left(D_{i}\right)$. and $L_{\delta_{\kappa}}\left(D_{j}\right)$ should be examined.

One approach to these complicated relations is conflict analysis [5]. In this analysis, several concepts which shares several attribute-value pairs, are analyzed with respect to qualitative similarity measure that can be viewed as an extension of rough inclusion. It will be our future work to introduce this methodology to analyze relations of boundary type and to develop an induction algorithms for these relations.

\section{References}

1. Adams RD and Victor M: Principles of Neurology, 5th edition. McGraw-Hill, New York, 1993.

2. Harris, J.M. Coronary Angiography and Its Complications - The Search for Risk Factors, Archives of Internal Medicine, 144, 337-341,1984.

3. Lin, T.Y. Fuzzy Partitions: Rough Set Theory, in Proceedings of Seventh International Conference on Information Processing and Management of Uncertainty in Knowledge-based Systems(IPMU'98), Paris, pp. 1167-1174, 1998.

4. Pawlak, Z., Rough Sets. Kluwer Academic Publishers, Dordrecht, 1991.

5. Pawlak, Z. Conflict analysis. In: Proceedings of the Fifth European Congress on Intelligent Techniques and Soft Computing (EUFIT'97), pp.1589-1591, Verlag Mainz, Aachen, 1997.

6. Pawlak, Z. Rough Modus Ponens. Proceedings of IPMU'98, Paris, 1998.

7. Polkowski, L. and Skowron, A.: Rough mereology: a new paradigm for approximate reasoning. Intern. J. Approx. Reasoning 15, 333-365, 1996.

8. Skowron, A. and Grzymala-Busse, J. From rough set theory to evidence theory. In: Yager, R., Fedrizzi, M. and Kacprzyk, J.(eds.) Advances in the Dempster-Shafer Theory of Evidence, pp.193-236, John Wiley \& Sons, New York, 1994.

9. Tsumoto, S. Automated Induction of Medical Expert System Rules from Clinical Databases based on Rough Set Theory Information Sciences 112, 67-84, 1998.

10. Tsumoto, S. Extraction of Experts' Decision Rules from Clinical Databases using Rough Set Model Journal of Intelligent Data Analysis, 2(3), 1998.

11. Tsumoto, S., Ziarko, W., Shan, N., Tanaka, H. Knowledge Discovery in Clinical Databases based on Variable Precision Rough Set Model. Proceedings of the Eighteenth Annual Symposium on Computer Applications in Medical Care, Journal of the American Medical Informatics Associations 2, supplement, pp.270-274,1995.

12. Tsumoto, S. 1999. Knowledge Discovery in Clinical Databases - An Experiment with Rule Induction and Statistics-In: Ras, Z.(ed.) Proceedings of the Eleventh International Symposium on Methodologies for Intelligent Systems (ISMIS'99), Springer Verlag (in press).

13. Zadeh, L.A., Toward a theory of fuzzy information granulation and its certainty in human reasoning and fuzzy logic. Fuzzy Sets and Systems 90, 111-127, 1997.

14. Ziarko, W., Variable Precision Rough Set Model. Journal of Computer and System Sciences. 46, 39-59, 1993. 